\title{
Honorés à l'égal des dieux et des héros ? La célébration des bienfaiteurs de la démocratie chez les orateurs attiques
}

Praised on a Par with Gods and Heroes? The Benefactors of the Democracy in the Attic Orators

\section{Sophie Gotteland}

\section{OpenEdition}

Journals

\section{Édition électronique}

URL : https://journals.openedition.org/mythos/2320

DOI : $10.4000 /$ mythos. 2320

ISSN : 2037-7746

\section{Éditeur}

Salvatore Sciascia Editore

\section{Référence électronique}

Sophie Gotteland, « Honorés à l'égal des dieux et des héros ? La célébration des bienfaiteurs de la démocratie chez les orateurs attiques », Mythos [En ligne], 14 | 2020, mis en ligne le 31 décembre 2020, consulté le 05 octobre 2021. URL : http://journals.openedition.org/mythos/2320 ; DOI : https:// doi.org/10.4000/mythos. 2320

Ce document a été généré automatiquement le 5 octobre 2021.

Mythos 


\title{
Honorés à l'égal des dieux et des héros ? La célébration des bienfaiteurs de la démocratie chez les orateurs attiques
}

\author{
Praised on a Par with Gods and Heroes? The Benefactors of the Democracy in \\ the Attic Orators
}

Sophie Gotteland

1 La place accordée aux exemples mythiques dans les discours attiques a depuis longtemps été relevée par la critique, et elle a fait l'objet de plusieurs études ${ }^{1}$. Sans craindre de tordre le matériau légendaire pour l'adapter à leur propos, les orateurs rappellent à de multiples reprises les exploits des héros grecs. Dans ce cadre rhétorique, les héros sont vantés avant tout pour leur force, leur vertu, leur courage, ou pour leur dévouement à la cause des Grecs. Ce sont les services qu'ils ont rendus à la communauté que l'on évoque, et les orateurs s'arrêtent peu sur ce qui distingue leur nature de celle des hommes, sur leur différence de statut avec eux et sur la dimension proprement religieuse attachée à ces figures ${ }^{2}$. L'objectif même des discours attiques, en effet, explique que l'on ne s'attarde pas sur les cultes et les honneurs qu'on leur rend. Les héros de l'épopée sont mis au service d'une parole politique qui exalte les valeurs de la cité démocratique ${ }^{3}$. Le rappel des exploits d'un Héraclès, d'un Thésée ou d'un Agamemnon doit avant tout susciter l'émulation chez les citoyens, leur $\zeta \tilde{\eta} \lambda$ o $\varsigma^{4}$, les inciter à égaler ces héros exemplaires dans l'espoir d'être célébrés à leur tour par le discours officiel de la cité comme les bienfaiteurs de la communauté.

Les orateurs n'hésitent pas, en revanche, à détailler les honneurs qui sont accordés aux citoyens pour prix de leurs services, et l'étude de leurs discours permet de retracer la hiérarchie de ces gratifications, qui vont de simples cadeaux jusqu'aux plus hautes récompenses comme l'érection d'une statue, l'entretien au prytanée et la proédrie. Pour certains citoyens, il semble même que puissent être institués après leur mort des honneurs exceptionnels, qui s'apparentent par bien des aspects à un culte et à une 
forme d'héroïsation. C'est le cas, notamment, pour les deux tyrannoctones, Harmodios et Aristogiton, ainsi que pour les soldats morts pour la patrie, à qui sont accordés des funérailles publiques au cours desquelles un orateur prononce une oraison funèbre. L'objectif de cet article est d'étudier précisément la nature de ces distinctions spécifiques et le vocabulaire qui sert à les dépeindre, pour tenter de déterminer le nouveau statut de ces individus. La cité, par la voix des orateurs, les fait-elle véritablement accéder à un nouvel état, entre immortalité et héroïsation, ou se contente-t-elle d'envisager ce changement de nature de manière métaphorique, pour mieux signifier sa reconnaissance et la gloire unique de ces individus? On envisagera aussi bien les discours d'apparat que les plaidoyers attiques, car ces discours, qui relèvent de deux types d'éloquence bien distincts, font malgré tout le même usage de ces figures, toujours sollicitées comme paradigmes d'un comportement exemplaire. Après avoir brièvement rappelé les récompenses auxquelles peuvent prétendre les citoyens pour prix de leurs bienfaits, on examinera dans un premier temps le cas d'Harmodios et d'Aristogiton dans les discours attiques avant d'envisager le sort réservé, selon les orateurs, aux citoyens morts à la guerre.

\section{Harmodios et Aristogiton en marche vers l'héroïsation}

3 Lorsqu'il énumère les différentes marques d'honneur dont peuvent bénéficier les hommes, Aristote cite indifféremment « les sacrifices, les inscriptions commémoratives en vers ou en prose, les privilèges, les attributions de terre, de places d'honneurs, les tombeaux, les statues, le fait d'être nourri aux frais de l'État, les usages barbares comme l'action de se prosterner ou de s'effacer devant quelqu'un -, les présents tenus pour précieux chez les uns ou les autres $»^{5}$.

4 Si elle n'apparaît pas explicitement ici, il y a bien pourtant une hiérarchie entre toutes ces marques d'honneur, et chacun des citoyens sait parfaitement le prix des $\mu \varepsilon ́ \gamma 1 \sigma \tau \alpha l$

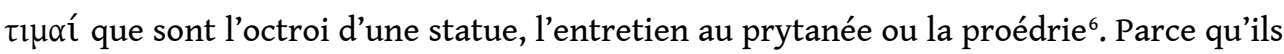
n'ont pas les mêmes objectifs que le philosophe, parce que la mention des présents octroyés à un citoyen leur sert à nourrir l'éloge de ce dernier, les orateurs soulignent explicitement, le plus souvent, les raisons qui justifient les honneurs particuliers dont certains bénéficient ; dans des cas rares, pour des bienfaits d'une nature exceptionnelle, ils suggèrent en outre que l'hommage de la patrie peut aller jusqu'à une forme d'héroïsation. Quand il célèbre les Grecs vainqueurs des Guerres médiques, Isocrate ébauche ainsi un rapprochement avec les héros mythiques, prétendant que grâce à leur valeur au combat, les soldats grecs ont obtenu la gloire d'être jugés dignes « des mêmes

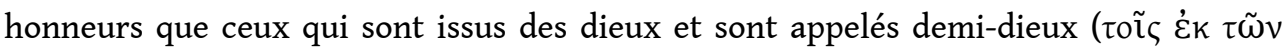

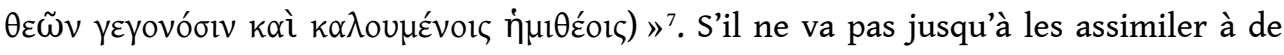
véritables héros (seul le souvenir de leur à petń est éternel ; leur nature, en revanche, ne connaît pas de changement), il bouscule malgré tout la frontière qui sépare pour nous temps mythiques et temps historiques en les gratifiant d'une forme d'immortalité ${ }^{8}$. Dans certains textes, il semble que les orateurs aillent plus loin encore et qu'ils suggèrent une proximité encore plus grande entre des citoyens exceptionnels et les héros de l'épopée.

5 Le cas d'Harmodios et d'Aristogiton est à cet égard remarquable, et il a suscité de nombreux commentaires chez les savants, qui ne s'accordent pas sur la nature exacte des honneurs qui leur sont rendus ${ }^{9}$. L'objectif n'est pas ici de trancher définitivement la 
question de l'héroïsation des tyrannoctones et de l'existence d'un culte à Athènes en leur honneur, mais d'étudier la position adoptée par les orateurs sur la question : de quelle manière présentent-ils les deux personnages, quel statut leur accordent-ils, et quels objectifs visent-ils à travers cette présentation? Leurs discours reviennent en effet à plusieurs reprises sur les honneurs exceptionnels qui leur sont attribués et sur ceux dont bénéficient également leurs descendants.

6 L'exploit des deux hommes est bien connu. Harmodios et Aristogiton sont célébrés pour avoir tué le tyran Hipparque, l'un des deux Pisistratides au pouvoir à Athènes, lors des Grandes Panathénées de 514. En punition de ce meurtre, ils sont arrêtés puis mis à mort. Dès l'Antiquité, les Anciens discutent toutefois leurs mobiles (geste politique ou vengeance amoureuse $?^{10}$ ) et le rôle effectif que les deux hommes ont joué dans la fin de la tyrannie, puisque leur action ne met pas immédiatement un terme à la tyrannie des Pisistratides : Hippias, le frère d'Hipparque, reste en vie ${ }^{11}$. Il faut attendre 510 pour qu'il soit renversé et pour qu'un régime démocratique soit progressivement mis en place à Athènes. Quoi qu'il en soit, la propagande démocratique s'empare très vite de cet épisode pour en faire un exploit fondateur pour la cité ${ }^{12}$. Harmodios et Aristogiton apparaissent alors comme les champions de la démocratie; ils sont célébrés comme ceux qui ont mis fin à la tyrannie, et des honneurs exceptionnels leur sont accordés, dont une statue en bronze sur l'Agora, érigée pour la première fois à cet endroit en l'honneur de bienfaiteurs de la patrie. Dans une cité qui répugne à accorder un statut et des honneurs particuliers à un individu, le choix d'honorer Harmodios et Aristogiton de cette manière montre bien la reconnaissance toute spéciale que leur vouent les Athéniens ${ }^{13}$. Pendant longtemps, nul n'a le droit d'ériger d'autres statues à côté de ce groupe de deux effigies, placé de manière emblématique devant le Portique de Zeus Eleutherios ${ }^{14}$. Les tyrannoctones disposent également d'un tombeau au Céramique ${ }^{15}$. On attribue enfin des privilèges exceptionnels à leurs descendants, dont la nourriture

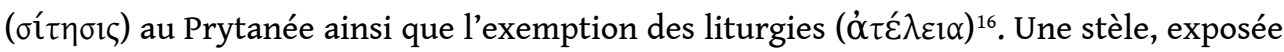
elle aussi sur l'Agora, rappelle à tous ces privilèges ${ }^{17}$.

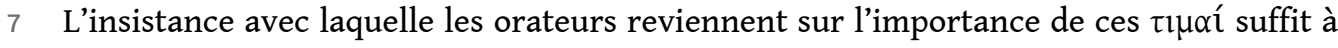
faire comprendre la valeur des bienfaits dont ils créditent Harmodios et Aristogiton ${ }^{18}$. Dans leurs discours, Harmodios et Aristogiton sont ainsi utilisés comme topos lorsqu'il s'agit d'évoquer un citoyen bienfaiteur de la démocratie. Leur renommée n'est jamais remise en question et ils apparaissent comme l'étalon à l'aune duquel on mesure les prouesses de tel ou tel individu et la reconnaissance qui lui est due. Très souvent, ils sont simplement évoqués pour leur exploit, sans précision supplémentaire sur les honneurs qui leur sont rendus, ou pour rappeler les privilèges dont jouissent leurs

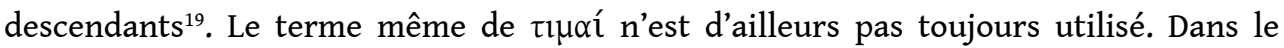
Contre Midias, Démosthène rappelle aux Athéniens qu'Harmodios et Aristogiton «ont reçu [d'eux] les plus grandes récompenses ( $\left.\mu \varepsilon ́ \gamma ı \tau \tau \alpha \_~ \delta \omega \rho \varepsilon \imath \alpha i\right)$, pour les plus grands services $»^{20}$, modifiant ainsi l'expression $\mu \varepsilon ́ \gamma \mid \sigma \tau \alpha l \tau \imath \mu \alpha i ́$ pour introduire un rapport qui semble moins officiel, plus personnel, entre les Athéniens et les tyrannoctones.

Certains discours se montrent néanmoins plus précis. Ainsi Démosthène, dans le Contre Leptine, évoque les différents privilèges dont jouissent les tyrannoctones et leurs descendants. Dans ce discours, daté de 355/354, l'orateur s'oppose à la réforme proposée par Leptine. Ce dernier souhaite en effet qu'Athènes revienne sur les privilèges dont bénéficient les différents bienfaiteurs athéniens, notamment l'exemption des liturgies. Pour faire mieux apparaître l'injustice d'une telle 
proposition, Démosthène rappelle les noms de plusieurs citoyens ainsi récompensés pour leur dévouement à la démocratie. Il cite entre autres le cas du stratège Conon, et souligne les récompenses exceptionnelles dont ce dernier a été gratifié après sa victoire de Cnide en 394 :

C'est pourquoi non seulement les hommes d'alors lui accordèrent l'exemption, mais ils lui érigèrent aussi une statue de bronze, le premier à en bénéficier depuis

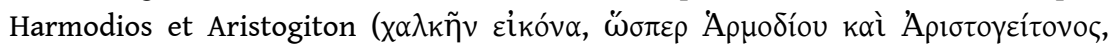
Ě $\sigma \tau \sigma \alpha v \pi \rho \omega ́ \tau \sigma o v)$. Ils pensaient en effet qu'il avait mis fin lui aussi à une lourde tyrannie en détruisant la puissance lacédémonienne. ${ }^{21}$

L'orateur prend soin d'insister sur le caractère unique de la faveur accordée à Conon, et sur la nouveauté du phénomène ${ }^{22}$. Il justifie un tel traitement pour le stratège en rapprochant explicitement les exploits de Conon, qui a restauré les longs Murs d'Athènes après la victoire de Cnide, et celui des deux tyrannoctones. Il en va dans un cas comme dans l'autre du salut de la démocratie et de sa victoire sur la tyrannie. C'est le mobile de cet acte, très clairement, qui explique que les Athéniens introduisent un tel changement dans leurs pratiques ${ }^{23}$. Le rapprochement montre bien le regard très particulier que les Grecs portent sur les tyrannoctones, dont la présentation comme étalon d'héroïsme et d'exploits marque une étape dans l'histoire de la démocratie.

Un passage du discours de Démosthène Sur l'ambassade, toutefois, signale explicitement des éléments d'un culte rendu aux deux tyrannicides. Alors que l'orateur évoque le sort de certains ambassadeurs athéniens, condamnés à un lourd châtiment par la cité quand bien même leurs comportements étaient beaucoup moins répréhensibles que celui d'Eschine lors de son ambassade auprès de Philippe II de Macédoine en 346, il en vient à citer, entre autres victimes, un descendant d'Harmodios. Le rappel des services exceptionnels rendus par ses ancêtres est un moyen de renforcer le contraste et de mieux souligner le scandale de cette injustice.

Eh bien, Athéniens, puisque les choses sont ainsi, vous, qui êtes les fils de ces hommes-là, et ceux d'entre eux, aussi, qui sont encore vivants -, vous supporterez que le bienfaiteur du peuple, l'homme qui est revenu du Pirée, Épikratès, ait été chassé et puni? que tout récemment, le fameux Thrasybule, fils du démocrate Thrasybule qui a ramené le peuple de Phylè, ait dû payer une amende de dix talents? que le descendant d'Harmodios et de nos plus grands bienfaiteurs, que vous associez, par la loi, aux offrandes et aux libations de vin lors des sacrifices dans tous vos sanctuaires en raison des bienfaits qu'ils nous ont rendus, que vous

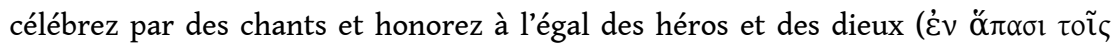

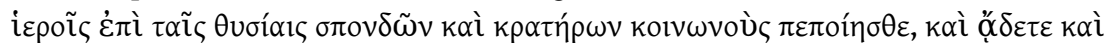

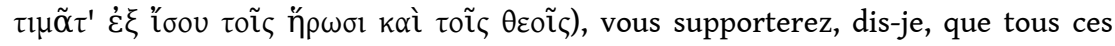
gens-là aient subi la peine fixée par les lois [...] ? ${ }^{24}$

11 Il importe peu que le nom de ce descendant des tyrannoctones ne soit pas donné25, ni que le seul Harmodios soit ici nommé, associé de manière large à l'ensemble des membres de sa famille ${ }^{26}$, et non spécifiquement à son compagnon Aristogiton. La citation, en revanche, semble relier les tyrannoctones, par le biais de trois éléments, à une forme de célébration rituelle inscrite dans un cadre religieux. L'orateur mentionne tout d'abord les offrandes et les libations ${ }^{27}$ accordées dans un espace consacré ( $\dot{\varepsilon} v$

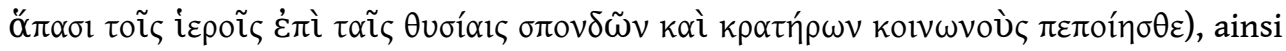
que la nature officielle de cette célébration, reconnue par une loi ( $v$ ó $\omega)$. Le détail des célébrations, qui se déroulaient peut-être lors des Panathénées ${ }^{28}$, n'est malheureusement pas donné, mais les circonstances dans lesquelles elles se déroulent 
ainsi que la forme qu'elles prennent nous placent sans ambiguïté dans un contexte religieux.

Un passage de la Constitution des Athéniens d'Aristote apporte apparemment une confirmation de ces pratiques. Parmi les charges religieuses qui relèvent du polémarque, le philosophe cite les points suivants :

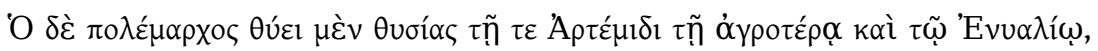

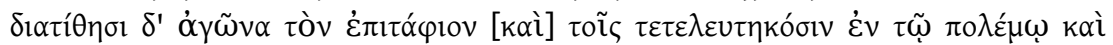

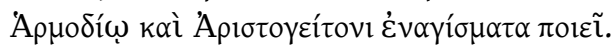

Le polémarque est chargé des sacrifices à Artémis chasseresse et à Enyalios, il organise les jeux funéraires pour ceux qui sont morts à la guerre et il offre les sacrifices funéraires en l'honneur d'Harmodios et d'Aristogiton. ${ }^{29}$

Le texte confirme qu'il y a bien un culte en l'honneur des tyrannoctones, même si le

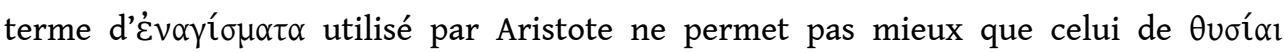
employé par Démosthène d'envisager plus précisément en quoi consistait le rituel ${ }^{30}$. Pour Gunnel Ekroth, il est très probable qu'il comprenait le sacrifice d'un animal, qui était brûlé dans sa totalité au cours de la cérémonie ${ }^{31}$.

Démosthène mentionne un deuxième élément contribuant à la célébration des tyrannoctones, les chants en leur honneur. Avec le verbe ợ $\delta \varepsilon \tau \varepsilon, ~ l ' o r a t e u r$ fait sans doute allusion aux diverses chansons à boire célébrant les deux héros, qu'Aristophane mentionne à plusieurs reprises, témoignant par là-même de la popularité de ces skolia à son époque, et que nous transmet également Athénée ${ }^{32}$. Or ces chansons elles aussi, pour l'une d'entre elles au moins, semblent associer les deux hommes à des héros :

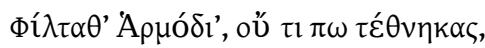

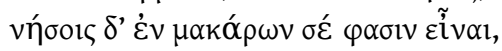

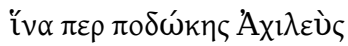

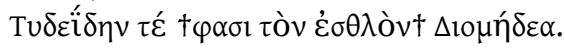

Très cher Harmodios, non, tu n'es pas mort du tout,

mais on dit que tu te trouves dans les îles des Bienheureux,

là où vit Achille aux pieds légers

et, dit-on, le noble Diomède, fils de Tydée. ${ }^{33}$

Dans ce skolion, le lieu du séjour d'Harmodios et Aristogiton (les îles des Bienheureux) ainsi que leur association à des héros (Achille et Diomède, en l'occurrence) montre suffisamment qu'ils occupent une place particulière dans l'imaginaire athénien. Ce rapprochement avec des figures épiques reconnues correspond d'ailleurs à ce que l'on trouve dans le Contre Timarque d'Eschine, lorsque l'orateur et ses adversaires évoquent des couples de jeunes garçons célébrés par la cité et les poètes pour la qualité de l'amour noble qui les lie. Là encore, le premier exemple cité est celui des tyrannoctones, mentionnés juste avant la paire formée par Patrocle et Achille ${ }^{34}$, et cet artifice littéraire est une autre façon de leur faire quitter la sphère historique pour les associer à la gloire des temps légendaires, de suggérer leur identité de statut avec des figures mythiques.

16 Le dernier élément souligné par le discours Sur l'ambassade confirme de manière plus explicite encore ce rapprochement. Harmodios et Aristogiton, affirme l'orateur, sont honorés par les Athéniens "à l'égal des dieux et des héros ». Une fois encore, le verbe utilisé par Démosthène ( $\tau 1 \mu \tilde{\tau} \tau \varepsilon)$ est peu précis, et ne nous permet pas d'envisager réellement quelles marques d'honneur les Athéniens leur réservaient ${ }^{35}$. Il est toutefois fréquemment utilisé à l'époque classique pour évoquer les honneurs rendus à un dieu 
ou un héros dans le cadre d'un culte, et on peut ainsi concevoir qu'il prenne cette valeur dans notre passage ${ }^{36}$.

Tout un faisceau d'indices pointe donc vers l'existence d'un culte héroïque en l'honneur d'Harmodios et d'Aristogiton à l'époque classique. Pour certains chercheurs,

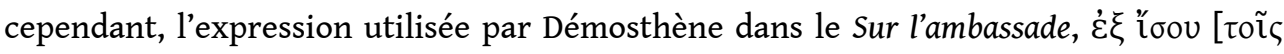

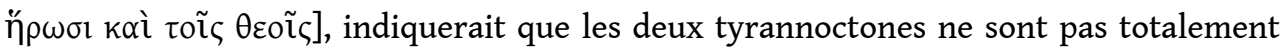
assimilés par l'orateur à des dieux ou des héros ${ }^{37}$. L'expression, en effet, semble suggérer qu'ils relèvent d'une catégorie à part, proche par bien des aspects des deux premières sans qu'ils en partagent malgré tout complètement la nature. Ils sont honorés autant que les dieux et les héros, comme les dieux et les héros (par le culte qui leur est rendu, par leur lieu de séjour), mais il n'y a pas complète identification avec eux. On peut cependant interpréter différemment ce $\varepsilon \dot{\xi} \zeta$ ľ $\sigma o v$, et penser qu'il a moins pour fonction, chez Démosthène, de souligner la différence entre les deux catégories évoquées que la volonté de rapprochement entre elles. Les chansons qui célèbrent Harmodios et Aristogiton, les discours qui les évoquent, montrent en effet qu'il existe à l'époque classique, dans la mentalité collective, la volonté de leur attribuer un statut particulier, de les distinguer des simples citoyens pour en faire des héros démocratiques. Cela passe par l'érection d'une statue, la création d'un culte et la diffusion de chansons les exaltant aux côtés des plus grands héros épiques. Des orateurs comme Démosthène et Eschine participent pleinement à cette « héroïsation » politique des deux hommes qui, dans le discours officiel de la cité, finissent par être transfigurés et par quitter la sphère historique pour acquérir une gloire les rapprochant toujours davantage des héros épiques, avant de finir, plus tard, par être véritablement assimilés à des héros ou même à des divinités ${ }^{38}$. Leur exploit au service de la cité justifie un tel traitement.

\section{Les guerriers morts pour la patrie : à la recherche d'un nouveau poste}

Lorsqu'Aristote, dans la Constitution des Athéniens, évoque les honneurs accordés par la cité à Harmodios et Aristogiton, il mentionne également à leurs côtés le sort particulier fait à ceux qui sont morts à la guerre, pour qui sont organisés des jeux et peut-être également, selon le texte que l'on adopte, des sacrifices funéraires ${ }^{39}$. Parce qu'ils ont eux aussi lutté pour défendre la liberté de la cité, les soldats morts au combat reçoivent en effet, lors de leurs funérailles, un hommage exceptionnel de la cité dans le cadre d'une cérémonie où Athènes rend hommage à leur courage. Les oraisons funèbres composées par les orateurs offrent un témoignage précieux sur ces pratiques ${ }^{40}$. Or, dans la manière dont les orateurs présentent le destin des guerriers morts au combat, il nous semble possible de sentir une évolution vers une forme de plus en plus affirmée d'héroïsation. Choisi par la cité pour prononcer l'éloge des morts à Chéronée, Démosthène rappelle ainsi les privilèges dont jouissent ces guerriers :

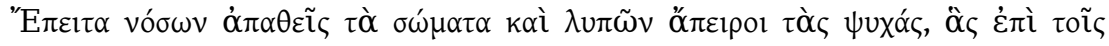

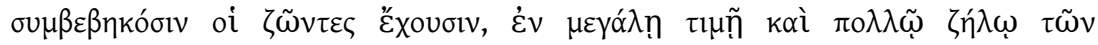

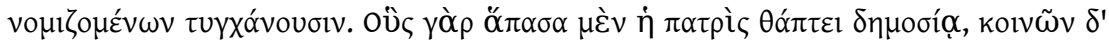

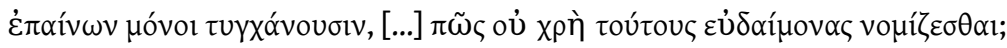

Ensuite, épargnés par des maladies physiques, à l'abri des peines de l'âme, que subissent les vivants au gré des événements, ils obtiennent les honneurs traditionnels en étant l'objet d'une grande estime et d'une profonde émulation. 
Ceux à qui la cité tout entière offre des funérailles publiques, qui sont les seuls à

obtenir des éloges communs, [...] comment ne pas les juger heureux ? $^{41}$

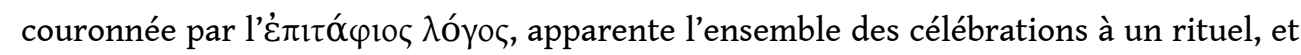
suggère des comportements codifiés par la tradition ${ }^{42}$. À cela s'ajoute, comme précise l'orateur un peu plus loin, la faveur insigne que les morts soient «jugés dignes de

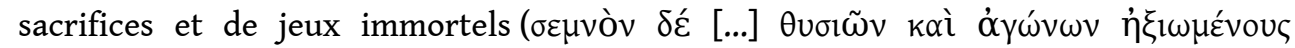

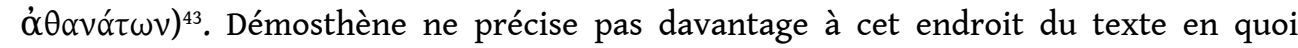
consistent ces jeux offerts en l'honneur des guerriers morts, mais il fait sans doute allusion à leur contenu un peu plus haut dans le texte, lorsqu'il demande la bienveillance de son auditoire : il a été désigné pour prononcer un discours, explique-til, non « pour honorer ces funérailles par une dépense pécuniaire, ou encore par un spectacle de concours hippiques ou gymniques (

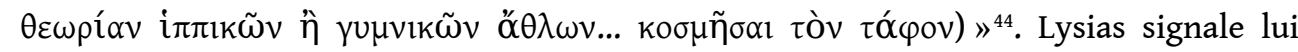
aussi, à côté de la cérémonie des funérailles publiques pour les guerriers morts, la tenue «en leur honneur de jeux de force, de sagesse et de richesse ( $\dot{\alpha} \gamma \tilde{\omega} v \varepsilon \varsigma$ [...] $\dot{\varepsilon} \pi^{\prime}$

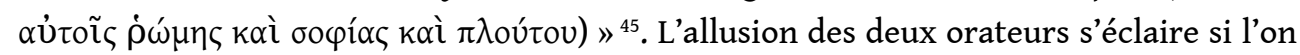
rapproche leurs discours d'un passage du Ménexène de Platon. Bien qu'il s'agisse de l'œuvre d'un philosophe, ce dialogue se présente comme une parodie des oraisons funèbres et mérite, à ce titre, d'être examiné en même temps que le texte des orateurs. Socrate y rapporte le discours qu'Aspasie aurait prononcé la veille devant lui pour lui montrer ce qu'il convient de dire en de telles circonstances. La jeune femme précise entre autres, dans son oraison funèbre, le rôle de la cité dans cette célébration officielle des soldats disparus :

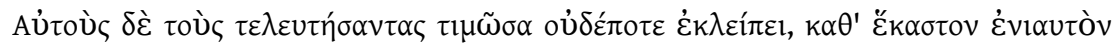

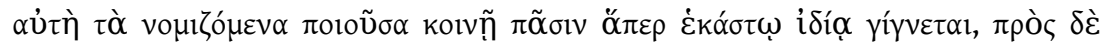

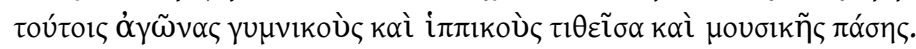

Aux morts eux-mêmes, [la cité] ne cesse jamais de rendre hommage : chaque année, c'est elle qui organise pour tous en public les cérémonies traditionnelles que l'on célèbre pour chacun en particulier; elle y ajoute des jeux gymniques et hippiques ainsi que des concours musicaux de toute nature. ${ }^{46}$

20

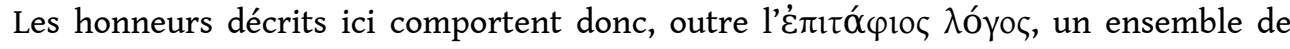
jeux funéraires dans lesquels on peut être tenté de retrouver les trois catégories d'ỏ $\gamma \omega ́ v$ indiquées par Lysias. Aspasie évoque également, un peu avant dans son texte,

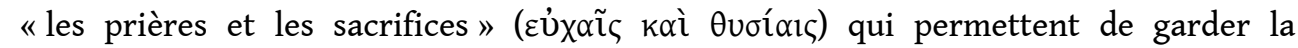
mémoire des morts $^{47}$. On retrouve ainsi dans cette parodie d'oraison funèbre les différents éléments d'un rituel dont la périodicité est ici explicitement signalée et qui est une nouvelle fois désigné par l'expression déjà relevée chez Démosthène, tò

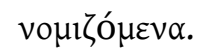

21 Comme l'a signalé Nicole Loraux, toutefois, le texte des oraisons funèbres ne lie pas explicitement la cérémonie des funérailles publiques, durant laquelle l'orateur

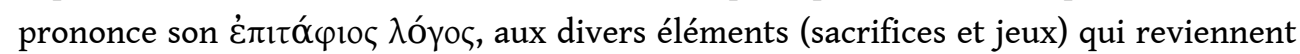
annuellement et marquent le culte rendu aux morts. Lysias, notamment, " associe et distingue tout à la fois les funérailles et les concours $\aleph^{48}$ quand il précise que la cité « fait des funérailles publiques aux soldats morts et [qu']elle organise pour eux des jeux

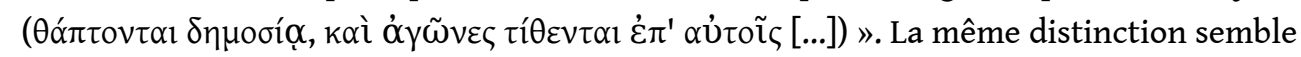
opérée par Platon dans le passage du Ménexène que nous venons de citer, puisqu'il use de deux participes ( $\pi \circ 10 \tilde{\sigma} \sigma \alpha$ et $\tau \imath \theta \varepsilon \tilde{\tau} \sigma \alpha$ ), reliés par une particule connective augmenté 


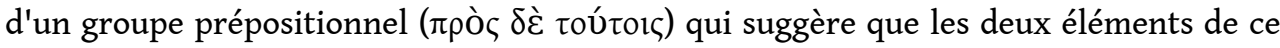
rituel de célébration ne sont pas forcément concomitants ${ }^{49}$.

S'il paraît donc avéré qu'il existe un culte en l'honneur des guerriers d'Athènes et que ce culte comprend un ensemble de prières, de sacrifices et de jeux, un certain flou entoure le statut dont les guerriers bénéficiaient. Étaient-ils considérés comme de véritables héros? Sur ce point, les textes des orateurs ne sont pas explicites; il semble bien, en outre, qu'ils n'adoptent pas tous la même position et qu'on puisse déceler, au cours du IV siècle, une évolution vers une forme de plus en plus assumée d'héroïsation.

Chez Lysias, qui prononce son Oraison funèbre à une date qui oscille entre 392 et 386, la seule immortalité offerte aux guerriers est celle de la mémoire et des honneurs. L'orateur martèle que tous les hommes sont mortels et que la mort est la même pour tous, qu'ils soient les meilleurs ou les pires des hommes ${ }^{50}$. La fin du discours est plus

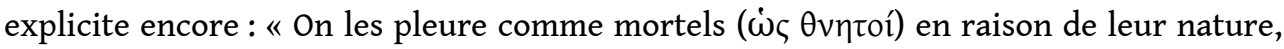
mais on les chante comme immortels ( $\dot{\omega} \varsigma \dot{\alpha} \theta \alpha \dot{v} v \alpha \tau o l)$ en raison de leur valeur $»^{51}$. S'ils

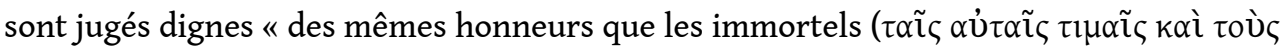

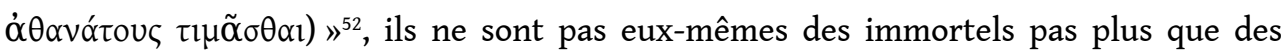

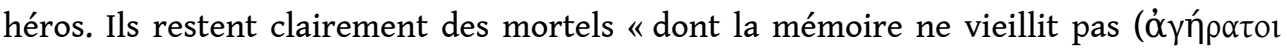

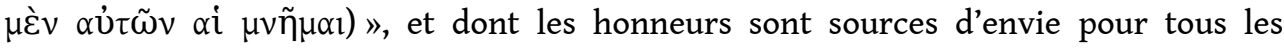
hommes ${ }^{53}$.

On retrouve chez Démosthène, dont l'Epitaphios date de 338, l'idée d'une " gloire qui ne

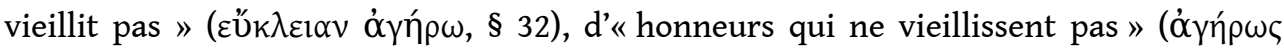

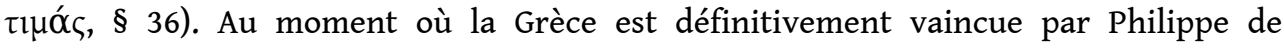
Macédoine, il est important d'affirmer la permanence, par delà la défaite, de la réputation et des valeurs grecques. Mais le statut des soldats morts, «épargnés par les maladies et les chagrins $"^{54}$, est chez lui beaucoup plus ambigu :

Ceux à qui la cité tout entière offre des funérailles publiques, qui sont les seuls à obtenir des éloges communs, [...] comment ne pas les juger heureux ? On pourrait dire, avec vraisemblance, qu'ils sont assis aux côtés des dieux d'en bas, partageant avec les braves qui les ont précédés le même rang dans les îles des Bienheureux

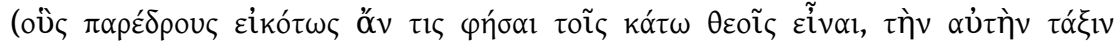

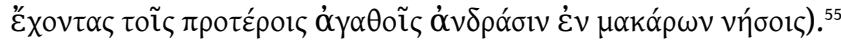

Là encore, le mot de « héros » n'est pas prononcé, mais la description par l'orateur du destin accordé aux soldats après leur mort évoque un tel statut, à la fois par le lieu de leur séjour et par la place d'honneur qui leur est accordée, aux côtés des dieux chtoniens. Cette présentation constitue pour Démosthène une reconstruction «vraisemblable» ( $\varepsilon \dot{i} \kappa o ́ \tau \omega \varsigma)$. Il ne s'aventure pas plus loin. Mais il affirme qu'il est

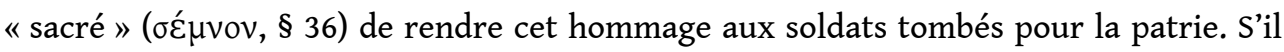
est vrai, comme il l'explique plus haut dans son discours, que le temps transforme peu à peu les exploits des hommes en mythes et qu'il les élève peu à peu à la dignité héroïque ${ }^{56}$, il inscrit ici les morts dans une chronologie qui doit progressivement leur permettre d'accéder à ce statut. Ils nourrissent la mythologie de la cité, servent d'exemples pour les citoyens et la remémoration de leurs exploits, lors de cérémonies publiques qui réunissent toute la cité, sert à leur donner, aux yeux des autres citoyens, ce statut spécial qui permet de les assimiler progressivement à des héros.

Hypéride, qui prononce son Oraison funèbre au printemps 322, en l'honneur des Athéniens morts durant la première année de la Guerre Lamiaque "pour la défense de la Grèce $~^{57}$, va plus loin que ses prédécesseurs. Son discours signale explicitement un 
changement de statut pour les soldats qu'elle célèbre. Comme Lysias ou Démosthène, Hypéride affirme, certes, que ces valeureux guerriers obtiendront en toute circonstance et en tout lieu émulation et éloges les plus grands (§ 30$)$. Mais il refuse pour sa part d'affirmer qu'ils sont morts et, usant d'une métaphore militaire, prétend

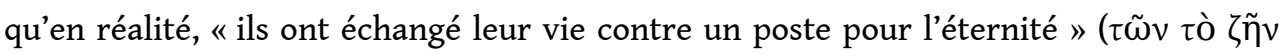

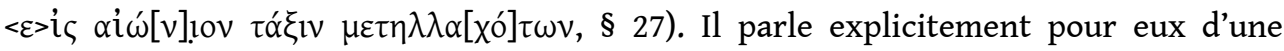
« seconde naissance » :

Comment, à bon droit, ne pas juger qu'ils sont favorisés par la fortune, estimer qu'ils ont cessé de vivre et non pas qu'ils ont obtenu une seconde naissance, plus

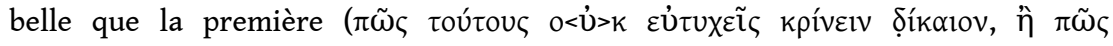

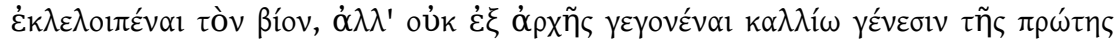

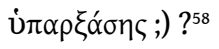

Les soldats célébrés échappent donc à la mort et gagnent l'éternité, obtenant de la sorte la qualité qui les distingue définitivement des mortels. La suite du discours offre d'autres indices confirmant cette lecture. Ainsi, lorsque l'orateur évoque le séjour du général Léosthène dans l'au-delà, il imagine qu'il sera accueilli par ceux qui ont combattu à Troie, et jouant sur un topos bien connu, prétend qu'il a surpassé ceux que l'on qualifie de «demi-dieux» $(\tau \tilde{\omega} v<\dot{\eta} \mu \imath \theta \dot{\varepsilon}>\omega v \quad \kappa \alpha \lambda \circ v \mu \varepsilon \dot{\varepsilon} v<\omega v>)^{59}$. Il termine sa description en suggérant que le stratège vainqueur résidera dans l'Hadès aux côtés d'Harmodios et d'Aristogiton ${ }^{60}$. Ce compagnonnage dans l'au-delà avec les deux tyrannoctones, dont les bienfaits sont reconnus par tous les Athéniens et qui font l'objet d'un culte rituel, comme on l'a vu plus haut, ne peut être ici anodin. Le rapprochement s'explique par leur combat commun pour la liberté grecque contre toute forme de tyrannie (39-40). Même si l'orateur, dans les dernières lignes qui nous sont parvenues de ce discours mutilé, se montre prudent et se contente d'affirmer lui aussi qu'il est vraisemblable ( $\varepsilon \dot{i} \kappa o ́ \varsigma)$ que les guerriers morts « obtiennent de la divinité la plus grande sollicitude et la plus grande attention $»^{61}$, sans s'avancer plus avant, de nombreux éléments, dans son discours, suggèrent qu'il tient à présenter les soldats morts comme bénéficiant d'un statut particulier correspondant, par bien des aspects, à celui des héros.

La présentation adoptée ici par Hypéride tranche avec la vraie hostilité dont il témoigne peu avant dans son discours, lorsqu'il évoque les honneurs divins ou héroïques accordés à Alexandre et à son compagnon Héphestion par les Athéniens à la demande du roi de Macédoine. Au paragraphe 21, il critique violemment les prétentions d'Alexandre et s'emporte contre ceux qui cherchent à brouiller indûment, sans le mériter, les frontières entre hommes et dieux ${ }^{62}$ :

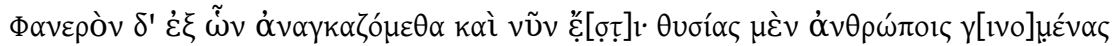

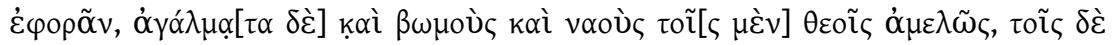

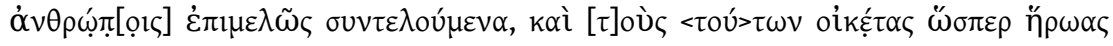

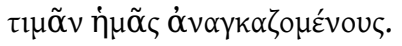

Cela est clair d'après ce à quoi nous sommes contraints et ce qui se passe en ce moment : voir des sacrifices effectués en l'honneur de simples hommes, ainsi que des statues, des autels et des temples célébrés pour les dieux sans empressement, mais pour les hommes avec empressement, et nous, contraints d'honorer les serviteurs de ces gens-là comme des héros.

Dans ce passage, l'orateur dénonce une perversion des pratiques cultuelles destinées aux dieux, et la reprise des différents éléments qui marquent le culte d'une divinité (sacrifices, temples, autels et statues) vient souligner le scandale du traitement qu'exige Alexandre de Macédoine $^{63}$. Hypéride pointe en outre avec ironie la reproduction, dans 
ce cadre rituel, de la hiérarchie qui existe dans le cadre politique : Alexandre se voit accorder les honneurs d'un dieu, mais son serviteur, pour sa part, ne peut prétendre qu'au traitement réservé à des héros. Comme l'a bien souligné Gunnel Ekroth, la

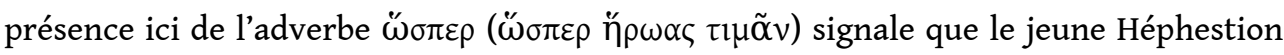
ne peut être véritablement considéré comme un héros, et que c'est en toute injustice qu'il demande à être traité comme tel ${ }^{64}$. Quand bien même les éléments de ce culte ne sont pas détaillés (on retrouve ici les mêmes termes généraux de $\theta v \sigma i ́ \alpha 1$ et de $\tau \imath \mu \tilde{\alpha} v$ que dans les exemples précédents), l'orateur veut signifier qu'il y a dans ce cas-là une appropriation abusive des signes et des honneurs rendus aux dieux et aux héros. Or, à l'inverse de Léosthène et de ses hommes ou des tyrannoctones, Alexandre ne mérite aucune admiration particulière, lui qui est présenté dans tout le passage comme une menace pour la liberté des Grecs. Les exigences du roi sont là pour révéler l'insolence

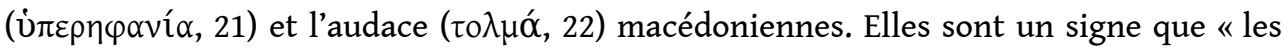
lois divines ont été abolies». Alexandre ne peut en effet prétendre aux mêmes honneurs à Athènes que ceux qui se sont battus pour la démocratie et pour les valeurs dont la cité se fait le champion. Le contraste est censé souligner le scandale des prétentions d'Alexandre.

La présence conjointe, à l'intérieur d'un même discours, de positions si divergentes concernant l'héroïsation d'individus par la cité est en soi intéressante. Elle reflète les pratiques nouvelles qui marquent cette époque et les débats suscités par ces changements au sein de la cité. Comme le montre l'étude des discours attiques, les orateurs n'assimilent jamais explicitement les plus grands bienfaiteurs de leur cité à des héros. Ils décrivent des pratiques rituelles, rappellent les cultes et les sacrifices qui honorent certains d'entre eux, insistent sur tous les traits qui les rapprochent des divinités et des figures mythiques, mais semblent toujours reculer lorsqu'il s'agit de les qualifier de héros. Il n'est sans doute pas anodin que les formulations plus explicites que l'on trouve chez Démosthène ou Hypéride interviennent à une période où l'oraison funèbre choisit de glorifier individuellement le stratège mort aux côtés des soldats, à un moment où les stratèges peuvent espérer obtenir, comme Conon, Iphicrate, Chabrias, l'honneur exceptionnel d'une statue sur l'Agora, et où la glorification de l'individu, et non plus de la seule communauté des citoyens, semble devenue plus acceptable. Dans un tel contexte, les bienfaiteurs de la démocratie fournissent aux orateurs des exemples plus persuasifs encore pour leurs concitoyens que les héros de l'épopée dont ils semblent partager tant de traits. Comme l'affirme Démosthène, le temps, peu à peu, se chargera de les faire passer définitivement du côté du mythe.

\section{BIBLIOGRAPHIE}

Azoulay 2014 : V. Azoulay, Les tyrannicides d'Athènes. Vie et mort de deux statues, Paris 2014.

CALAME 1996 : C. Calame, Thésée ou l'imaginaire athénien. Légende et culte en Grèce antique, Lausanne 1996. 
CHIRON 2007 : P. Chiron, Aristote. Rhétorique, traduction nouvelle, présentation, notes et index, Paris 2007.

Clairmont 1983 : C. W. Clairmont, Patrios Nomos. Public Burial in Athens during the Fifth and Fourth Century B.C. The Archaeological, Epigraphic-Literary, and Historical Evidence, 2 vols, Oxford 1983.

DAIX, FERnANDEZ 2017 : D.-A. Daix, M. Fernandez, Démosthène. Contre Aphobos I \& II, Contre Midias, Paris 2017.

EкROTH 2002 : G. Ekroth, The Sacrificial Rituals of Greek Hero-Cults in the Archaic to the Early Hellenistic Periods, Kernos, Suppl. 12, Liège 2002.

GAUTHIER, 1985 : Ph. Gauthier, Les cités grecques et leurs bienfaiteurs (IV -I $^{\text {er }}$ s. av. J.-C.). Contribution à l'histoire des institutions, Paris 1985.

GoTTELAND 2001 : S. Gotteland, Mythe et rhétorique. Les exemples mythiques dans le discours politique de l'Athènes classique, Paris 2001.

GoTTELAND 2012: S. Gotteland, «L'invention d'un héros paradigmatique : la figure de Conon chez les orateurs attiques ", in S. Dubel, S. Gotteland, E. Oudot (éds.), Éclats de littérature grecque d'Homère à Pascal Quignard. Mélanges offerts à Suzanne Saïd, Paris 2012, 63-87.

HERRMAN 2009 : J. Herrman, Hypereides. Funeral Oration, Oxford 2009.

HORNBLOWER 1991 : S. Hornblower, A Commentary on Thucydides, vol. I, books I-III, Oxford 1991.

JosT 1936 : K. Jost, Das Beispiel und Vorbild der Vorfahren bei den attischen Rednern und Geschichtschreibern bis auf Demosthenes (Rhetorische Studien 19), Paderborn 1936.

KEARNS 1989 : E. Kearns, The Heroes of Attica, BICS, Suppl. 57, London 1989.

Kimmel-ClaUzet 2013 : Fl. Kimmel-Clauzet, Morts, tombeaux et cultes des poètes, Bordeaux 2013.

KREMMYDAS 2012 : Chr. Kremmydas, Commentary on Demosthenes' Against Leptines, Oxford 2012.

LORAUX 1981 : N. Loraux, L'invention d'Athènes. Histoire de l'oraison funèbre dans la " cité classique », Paris - La Haye - New York 1981.

MACDOWELL 2000 : D. M. MacDowell, Demosthenes. On the False Embassy (Oration 19), edited with Introduction, Translation, and Commentary, Oxford 2000.

NOUHAUD 1982 : M. Nouhaud, L'utilisation de l'histoire par les orateurs attiques, Paris 1982.

PARKER 1996 : R. Parker, Athenian Religion. A History, Oxford 1996.

PAULSEN 1999 : Th. Paulsen, Die Parapresbeia-Reden des Demosthenes und des Aischines: Kommentar und Interpretation zu Demosthenes, or. XIX, und Aischines, or. II, Trier 1999.

PEARSON 1941 : L. Pearson, « Historical Allusions in the Attic Orators », Classical Philology 36 (1941), 209-229.

PERLMAN 1961 : S. Perlman, « The Historical Example, its Use and Importance as Political Propaganda in the Attic Orators ", Studies in History, Scripta Hierosolymitana 7 (1961), 150-166.

PRITCHETT 1985 : W. K. Pritchett, The Greek State at War, Part IV, Berkeley - London - Los Angeles 1985.

RHODES 1981 : P. J. Rhodes, A Commentary on the Aristotelian Athenaion Politeia, Oxford 1981.

RUDHARDT 1992 : J. Rudhardt, Notions fondamentales de la pensée religieuses et actes constitutifs du culte dans la Grèce classique, Paris $1992^{2}$. 
SCHMitz-KahlmanN 1939 : G. Schmitz-Kahlmann, Das Beispiel der Geschichte im politischen Denken des Isokrates (Philologus Suppl. Bd. 31, Heft 4), Leipzig 1939.

SHEAR 2007 :J. Shear, «Cultural Change, Space and the Politics of Commemoration in Athens », in R. Osborne (ed.), Debating the Athenian Cultural Revolution. Art, Literature, Philosophy, and Politics 430-380 B.C., Cambridge 2007, 91-115.

SHEAR 2012a : J. Shear, «Religion and the Polis. The Cult of the Tyrannicides at Athens », Kernos 25 (2012), 27-55.

SHEAR 2012b : J. Shear, « The Tyrannicides, their cult and the Panathenaia: a note ", Journal of Hellenic Studies 132 (2012), 107-119.

THOMPSON, WyCHERLEy 1972 : H. A. Thompson, R. E. Wycherley (eds.), The Agora of Athens. The Athenian Agora, vol. XIV: The History, Shape and Uses of an Ancient City Center, Princeton 1972.

VERSNEL 1995 : H. S. Versnel, « Religion and Democracy », in W. Eder (ed.), Die athenische Demokratie im 4. Jahrhundert v. Chr.: Vollendung oder Verfall einer Verfassungsform, Stuttgart 1995, 367-387.

WHITEHEAD, D. (2000) : D. Whitehead, Hypereides. The Forensic Speeches, Oxford 2000.

WYCHERLEY 1957 : R. E. Wycherley, The Agora of Athens. The Athenian Agora, vol. III: Literary and Epigraphical Testimonia, Princeton 1957.

\section{NOTES}

1. Entre autres, Jost 1936, Schmitz-Kahlmann, 1939, Pearson, 1941, Perlman, 1961, Nouhaud 1982 et GOTTELAND 2001.

2. Parmi les exceptions notables figurent les précisions apportées par Isocrate dans son Éloge d'Hélène $[\mathrm{X}]$ (61-63) sur le sort fait à Hélène, qui, par sa beauté, acquiert une puissance «égale à

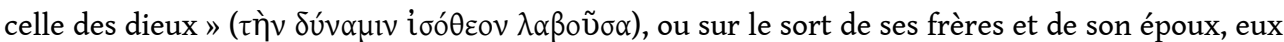
aussi introduits au rang de divinités. Le paragraphe 63 rappelle le culte consacré au couple d'Hélène et Ménélas à Thérapné, « non pas comme à des héros, mais comme à des dieux (oủx $\dot{\omega} \varsigma$

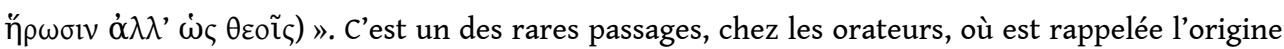
d'un culte (cf. RUDHARDT 1992, 131-132). Voir également Isocrate, Évagoras [IX], 15, sur le sort fait à Éaque, le fils de Zeus et l'ancêtre d'Évagoras, ainsi que Lycurgue, Contre Léocrate, 88, sur les privilèges accordés aux héros éponymes de l'Attique.

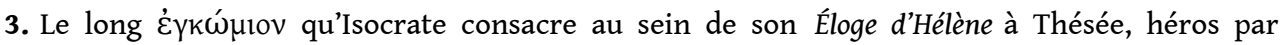
excellence d'Athènes, offre un très bon exemple de cette utilisation politique des exemples mythiques. L'orateur mentionne ses exploits contre les monstres ou les peuples barbares qui menacent Athènes et plus largement, la civilisation grecque, il s'attarde sur ses réformes politiques, mais il n'évoque jamais les différentes étapes d'une héroïsation marquée par le rapatriement de ses ossements de Skyros à Athènes vers 475, sous l'égide de Cimon, et par l'instauration d'un culte pour l'ancien roi d'Athènes, qui se voit attribué un monument officiel sur l'Agora, le Theseion. Il ne mentionne pas non plus les cultes et les pratiques rituelles que Thésée était censé avoir instaurés, ni les fêtes des Theseia, au cours desquelles il était honoré. Sur ces questions, voir CALAME 1996.

4. C'est encore plus vrai lorsque ces héros sont présentés comme les ancêtres de leur үर́voc. Voir Isocrate, Philippe [V], 76-77, 113-115,127, 132, Archidamos [VI], 8, ou encore Démosthène, Oraison funèbre [LX], 27-31.

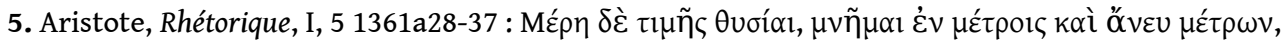

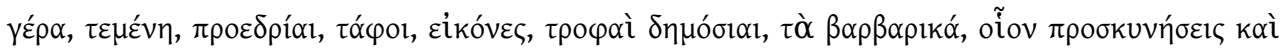




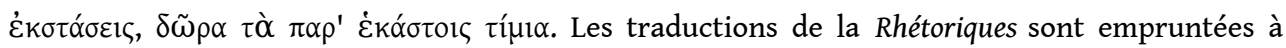
CHIRON 2007. Voir KimMEL-CLAUZET 2013, 192 pour l'analyse de ce passage.

6. Sur la mise en place de cette hiérarchie des honneurs, en haut de laquelle on trouve la triade

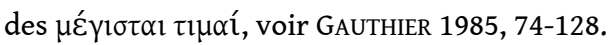

7. Isocrate, Panégyrique [IV], 84.

8. Sur les différentes stratégies linguistiques utilisées par les orateurs pour brouiller la frontière entre mythe et histoire, voir GOTTELAND 2001, 89-102.

9. Les deux tyrannoctones font l'objet d'une bibliographie imposante. On se contentera de citer ici les titres les plus intéressants pour notre étude : TAYLOR 1981, VERSNEL 1995, SHEAR 2007, SHEAR 2012a, SHEAR 2012b, Azoulay 2014. Comme le rappelle Vincent Azoulay, la plupart des chercheurs admettent que les tyrannoctones étaient les destinataires d'un culte héroïque. À la suite de certains autres, cependant, il choisit pour sa part de ne pas trancher en l'absence de preuves irréfutables (Azoulay 2014, 113-117).

10. Les deux versions concurrentes sont respectivement rapportées par Hérodote $(\mathrm{V}, 55)$ et par Thucydide (I, 20 et VI, 54-59). Cf. Aristote, Constitution d'Athènes, 18. Pour un bon résumé des divergences de présentation et des ambiguïtés d'interprétation, voir AzoulaY 2014, 29-37.

11. Si l'on en croit Hérodote (VI, 123 et V, 62), Thucydide (VI, 59, 2) et Aristote (Constitution d'Athènes, 19,1), il aurait même durci son régime à la suite de cet épisode.

12. On a pu ainsi parler d'une « reconstruction idéologique a posteriori » (DAIX, FERNANDEZ 2017, 414).

13. Ce sont effectivement, à cette époque, les premières et les seules statues honorifiques érigées sur l'agora pour des bienfaiteurs. Voir Démosthène, Contre Leptine [XX], 70, Aristote, Rhétorique, I, 9, 1368a et Pline, Histoires Naturelles, XXXIV, 17. Cf. SHEAR, 2007, 94, évoquant la période entre les années 430 et 420 : «Absent from the Agora were inscriptions and any statues other than the Tyrannicides ». Le premier groupe statuaire, exécuté par Anténor a priori après l'exil d'Hippias, en 510, fut emporté par Xerxès en 480 puis remplacé par un second groupe d'effigies, réalisées en 477/476 par Kritios et Nésiotès.

14. Pausanias 1.8.5. Cf. IG II ${ }^{2}, 450$, b7-12 et IG II $^{3} 1853$, 39-42. Pour l'ensemble des références, voir WYCHERLEY 1957, 93-98, et THOMPSON, WYCHERLEY 1972, 155-160.

15. Pausanias 1.29.15. Pour Gunnel Ekroth (ЕкRотн 2002, 84 n. 277), il est difficile de savoir si cet édifice était un véritable tombeau ou un simple cénotaphe, car on n'a pas de renseignements précis ce qu'il advint de leurs corps après leur mort.

16. Pour la nourriture au prytanée, voir IG I ${ }^{3} 131$, 5-7. Pour l'exemption des liturgies (et non des charges cultuelles), voir Démosthène, Contre Leptine [XX], 127-128.

17. Démosthène, Contre Leptine [XX], 170.

18. On trouvera les principales références commodément rassemblées dans NouHAUD 1982, 22 et KREMMYDAS 2012, 218-220.

19. Par exemple Isée, Succession de Dikaiogénès [V], 47, Hypéride, Contre Philippide [I], 2 et Démosthène, Contre Leptine [XX], 18, 29, 69-70, 127-128, 159-160.

20. Démosthène, Contre Midias [XXI], 170. Le changement s'explique dans un contexte où l'orateur stigmatise l'absence de générosité, de $\varphi \imath \lambda o \tau \iota \mu i ́ \alpha$, de son adversaire à l'égard de ses concitoyens.

21. Contre Leptine [XX], 70. Cf. id., 69, ce qui est gravé sur stèle : «Attendu que Conon a rendu la liberté aux alliés d'Athènes ".

22. D'autres exemples interviennent par la suite durant le $\mathrm{IV}^{\mathrm{e}}$ siècle, comme le regrette Démosthène (Contre Aristocrate [XXIII], 196-198 et Sur l'organisation financière [XIII], 21-22). Il semble qu'en réalité la pratique n'ait pas été si courante durant ce siècle. On connaît finalement peu de cas de stratèges honorés par une statue à cette époque : cela concerne Iphicrate, Chabrias, Timothée (Sur le détail des récompenses accordées à chacun d'eux, voir GAUTHIER 1985, 97-103). Pour le traitement réservé à Conon à cette occasion, voir GoTTELAND 2012, 13-14 et KREMMYDAS 
2012, 313-314. Dinarque, Contre Démosthène [I], 101, signale qu'une statue en bronze a été érigée pour Démade sur l'Agora, et il souligne lui aussi le caractère exceptionnel d'une telle récompense. Cf. encore Eschine, Contre Ctésiphon [III], 183-186.

23. Le parallèle était poussé encore plus loin car l'on sait que cette statue de Conon était placée près de celle d'Harmodios et d'Aristogiton. Les Athéniens, en outre, avaient dressé à côté de cette effigie de Conon une statue du roi Évagoras de Chypre, pour remercier ce dernier d'avoir aidé le stratège athénien dans sa lutte contre les Spartiates, créant ainsi un couple d'effigies en regard de celui d'Harmodios et d'Aristogiton (Isocrate, Évagoras [IX], 55-57). Lycurgue, Contre Léocrate, 51, confirme ce regard spécifique porté à Athènes sur les stratèges, qui protègent la démocratie et apparaissent ainsi comme les plus grands bienfaiteurs d'Athènes : "Seuls entre les Grecs vous savez, Athéniens, honorer les braves. Dans d'autres cités, ce sont les athlètes, vous vous en apercevrez, qui ont leur statue érigée sur l'agora, tandis que chez vous, ce sont de valeureux stratèges et les meurtriers des tyrans ».

24. Démosthène, Sur l'ambassade [XIX], 280-281. Sur les trois hommes évoqués par Démosthène et sur les faits qui ont entraîné leur condamnation, voir les précisions de MACDOWELL 2000, 324-326.

25. Pour beaucoup, à commencer par le scholiaste de Démosthène (Scholia demosthenica, 493, Dilts, vol II, p. 83), il s'agit du stratège Proxène, mentionné déjà à plusieurs reprises dans le discours $(50,52,73,154-155)$.

26. Comme le rappelle D. MacDowell (MACDowell 2000, 26), les tyrannoctones appartiennent à la famille des Géphyréens (Hérodote, V, 57).

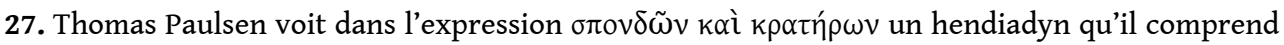
comme "des offrandes faites de libations de vin ", " des offrandes tirées des cratères de vin » (PAulSEN 1999, 265). Cf. ЕкRотн 2002, 179 n. 213. Comme l'a signalé R. Parker, il est difficile de comprendre à quoi correspondent exactement les libations auxquelles Démosthène fait ici allusion (PARKER 1996, 136 n. 55).

28. C'est en tout cas l'opinion de J. Shear, qui se fonde entre autres sur un passage de la Vie d'Apollonios de Tyane (VII, 4, 3) dans lequel Philostrate rappelle aux gouverneurs des provinces romaines «les Panathénées attiques, où l'on célèbre par des chants Harmodios et Aristogiton, ainsi que l'exploit de Phylè, qui a libéré d'un coup des trente Tyrans » (SHEAR 2012a, 32-35).

29. Aristote, Constitution des Athéniens, 58, 1. Le passage présente un problème de texte qui engage

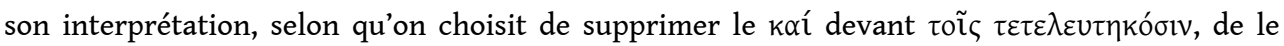
remplacer par غ̇ंí ou de le maintenir dans le texte. La première interrogation porte sur le fait de

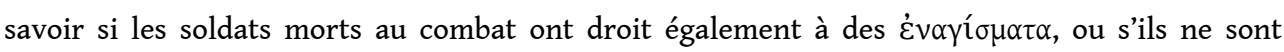
honorés que par des jeux funéraires; la seconde consiste à se demander si ces sacrifices funéraires, au cas où ils leur sont effectivement accordés, se déroulent en même temps pour eux et pour les tyrannoctones, ou s'il s'agit de sacrifices différents. Pour l'exposé des diverses positions, voir la discussion de RHODES 1981, 650-651 et d'EKROTH 2002, 83-85.

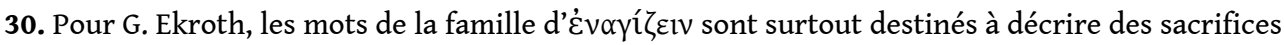
en l'honneur de personnes ou des héros décédés (ЕКRотH 2002, 233 ; 83-85, 170-171et 241 n. 130 pour le cas particulier des tyrannoctones). Pour elle, les $\dot{\varepsilon} v \alpha \gamma i ́ \sigma \mu \alpha \tau \alpha$ accomplis pour Harmodios et Aristogiton sont proches, mais pas identiques, au culte des guerriers morts à la guerre. Cf. RUDHARDT 1958, 238-239.

31. ЕKROTH 2002, 170-171 et 233-234. Cf. SHEAR 2012a, 30-32.

32. Voir n 893-896 PMG (= Athénée, Déipnosophistes, XV, 695a-b). Cf. les allusions nombreuses à l'un de ces skolia chez Aristophane, Acharniens, 980 et 1093, Guêpes, 1225sq., Lysistrata, 631sq. et Cigognes, fr. 444 Kassel-Austin. Hypéride, dans le Contre Phidippide, fr. 15b 4, rappelle qu'une loi interdisait de moquer les deux hommes dans des chansons.

33. $\mathrm{N}^{\circ} 896 \mathrm{PMG}$.

34. Eschine, Contre Timarque [I], 132-133. 


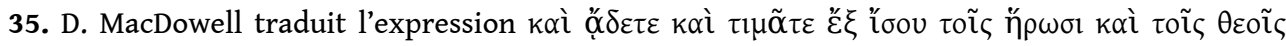
comme un hendyadin, rapprochant l'expression des skolia célébrant Harmodios et Aristogiton: les Athéniens « chantent les louanges » des tyrannoctones à l'égal des dieux et des héros (« whose praises you sing on par with the heroes and gods »).

36. Voir ЕKROTH 2002, 199-208 et KIMMEL-CLAUZET 2013, 192-193.

37. D. MacDowell reste prudent, mais penche pour cette position (MACDowELL 2000, 326). Pour R. Parker (PARKER 1996, 136-137), le terme de «héros " n'est jamais explicitement attribué aux tyrannoctones, et ils n'ont donc jamais, selon lui, été considérés comme des " héros » au sens propre. En faveur d'un culte héroïque en l'honneur des tyrannoctones, en revanche, on citera KeARNS 1989, 150, VeRSNEL 1995, 381-382, SHEAR 2012a 29-35. Cf. supra n. 9.

38. Cf. Cicéron, Pro Milone, 80 («Les Grecs décernent des honneurs divins aux meurtriers des tyrans ») et Valère Maxime, II, 10 (parlant de la statue des tyrans, que Séleucos fait rapporter en Grèce : «Les habitants de Rhodes, eux, quand elle arriva chez eux au cours de ce transport, lui offrirent l'hospitalité au nom de leur cité et la placèrent même sur un lit offert à la vénération »). 39. Voir supra, n. 29.

40. C'est Thucydide qui nous livre le premier compte-rendu, pour les sources littéraires, de cette cérémonie officielle, décrivant les différentes étapes des funérailles (exposition des corps,

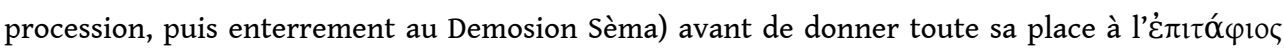
入óyos prononcé par Périclès (II, 34-47). La bibliographie sur l'oraison funèbre et, plus largement, sur la cérémonie des funérailles publiques à Athènes est impressionnante. Dans cet ensemble, voir LORAUX 1981, HORNBLOWER 1991 et HERMANN 2009 (avec les compléments bibliographiques). La date à laquelle cet usage a été instauré reste objet de discussion (sans doute à la fin des années 470, cf. HoRnBlower 1991, 292-293), de même que la périodicité, qui n'était sans doute pas annuelle au départ. On hésite également sur la période à laquelle se déroulait la cérémonie. Voir sur tous ces points LORAUX 1981, 38-39, PRITCHETT 1985, 106-124. HERMANN 2009, 14 n. 77, rassemble les principales références sur la question.

41. Démosthène, Oraison funèbre [LX], 33.

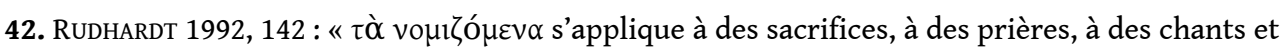
à des danses, à des purifications comme à l'ensemble de ce qui est dû aux dieux. C'est l'expression grecque la plus proche de notre mot "rite". [...] Ce sont des conduites imposées ou définies par des règles traditionnelles " .

43. Démosthène, Oraison funèbre [LX], 36.

44. Id., 13.

45. Lysias, Oraison funèbre [II], 80.

46. Platon, Ménexène, 249b (trad. de L. Méridier légèrement modifiée, Paris, CUF). Cf. 234c.

47. Id., 244a.

48. LORAUX 1981, 38. Elle précise juste après : «Tout indique donc que les morts reçoivent une double timè : honorés une fois pour toutes par des funérailles publiques et par un epitaphios, ils n'en sont pas moins tous les ans célébrés par un culte ». Mais PRITCHETT 1985, 129-130, considère que cette position est difficile à tenir. Pour lui, s'il y avait des jeux funéraires, comme l'attestent les témoignages littéraires, ils devaient se tenir au moment des funérailles publiques.

49. Respectivement Lysias, Oraison funèbre [II], 80 et Platon, Ménexène, $249 \mathrm{~b}$.

50. Lysias, Oraison funèbre [II], 77 : « Ignorions-nous que nous sommes tous mortels ? [...] Pourquoi cet accablement excessif devant un accident naturel, quand nous savons que les pires des humains sont comme les meilleurs devant la mort. [...] Elle est la même pour tous. »

51. Id., 80.

52. Ibid. 
53. Id., 79. De même le Ménexène de Platon, en $247 \mathrm{~d}$, prétend que les pères des soldats décédés, avant le départ de leurs fils au combat, ne prient pas pour que leurs enfants deviennent immortels, mais pour qu'ils deviennent braves et glorieux.

54. Démosthène, Oraison funèbre [LX], 33.

55. Id. 33-34.

56. Démosthène, Oraison funèbre [LX], 9 : «J'ai laissé de côté beaucoup d'exploits élevés au rang de mythes et j'ai rappelé seulement ceux-là [...]. En revanche, les exploits qui, pour le mérite, ne le cèdent en rien à ceux-là, mais qui, plus proches de nous dans le temps, n'ont pas encore fait l'objet de récits mythiques et n'ont été élevés au rang héroïque, voilà ce dont je vais désormais

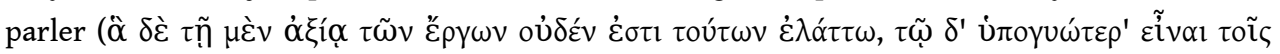

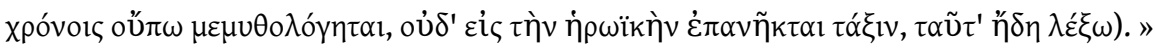
57. Hypéride, Oraison funèbre [VI], 17. Cf. $\$ 16$ : « pour la défense de la liberté des Grecs ».

58. Id., 27-28: le texte d'Hypéride présente différentes altérations, mais la plupart des éditeurs s'accordent sur les restitutions dans ce passage. L'édition adoptée ici est celle de Herrman, qui suit pour l'essentiel celle de Jensen (1917), tout en proposant sur un certain nombre de points des lectures différentes.

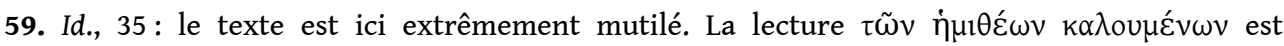

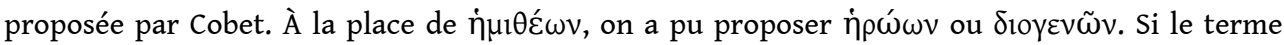
change, le référent reste toujours lié au monde des dieux.

60. Id., 39. Comme l'explique J. Herrman (HERRMAN 2009, 102-104), Hypéride va plus loin que les autres orateurs dans leurs oraisons funèbres puisqu'il prétend que Léosthène et ses hommes sont supérieurs aux héros de Troie et aux guerriers des guerres médiques. C'est en outre la seule oraison qui les compare à Harmodios et Aristogiton.

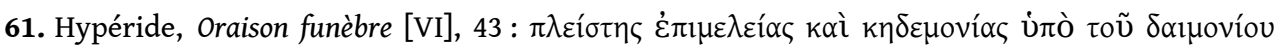

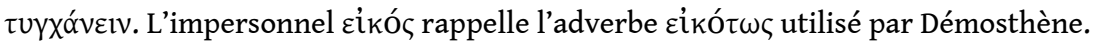

62. Les prétentions d'Alexandre datent sans doute de sa visite à l'oracle de Zeus Ammon dans l'oasis de Siwah, en 332. C'est seulement à partir de 324, semble-t-il, qu'il souhaite que ce statut lui soit reconnu également en Grèce. Voir HerRman 2009, ad loc., 88-90. Cf. Hypéride, Contre Démosthène [V], 31 (et les remarques de WHITEHEAD 2000, 455-457).

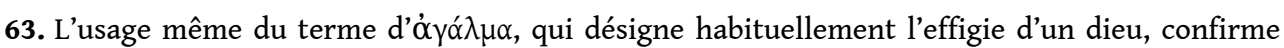

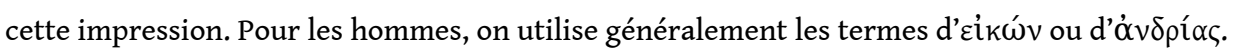

64. ЕКROTH 2002, p. 209-210.

\section{RÉSUMÉS}

Les orateurs attiques célèbrent abondamment les exploits des héros mythiques, figures exemplaires proposées à l'admiration des Athéniens, sans pour autant s'attarder sur les cultes et les sacrifices dont certains d'entre eux font l'objet au sein de l'espace civique. Pour inciter les Athéniens à égaler leurs hauts faits, les discours détaillent en revanche les honneurs qui récompensent les bienfaiteurs de la cité. Certains citoyens distingués par la cité pour leur dévouement à la démocratie jouissent en effet après leur mort d'honneurs exceptionnels qui s'apparentent par bien des aspects à un culte. On étudiera en particulier le cas d'Harmodios et d'Aristogiton ainsi que le sort réservé aux citoyens morts pour la patrie, qui reçoivent des honneurs spécifiques célébrés dans les oraisons funèbres. En analysant précisément ces honneurs 
et le vocabulaire qui sert à les dépeindre on tentera de déterminer le nouveau statut de ces individus, entre immortalité et héroïsation.

Attic orators extensively celebrate the deeds of mythical heroes, exemplary figures offered to the admiration of the Athenians, without dwelling on the cults and sacrifices devoted to them within the civic space. To encourage the Athenians to match their exploits, the speeches emphasize the honors rewarding the benefactors of the city. Some citizens indeed were distinguished by the city for their devotion to democracy and enjoyed after their death exceptional honors which are similar in many ways to a cult. We will study in particular the case of Harmodios and Aristogiton as well as the destiny reserved to citizens who died on the battle-field and received specific honors remembered by the Epitaphioi. By analyzing these honors and the vocabulary used to depict them, we will try to determine the new status of these individuals, between immortality and heroization.

INDEX

Mots-clés : orateurs, tyrannoctones, megistai timai, bienfaiteurs, oraison funèbre

Keywords : orators, Tyrannicides, megistai timai, benefactors, epitaphios logos

\author{
AUTEUR \\ SOPHIE GOTTELAND \\ Université Bordeaux Montaigne - Institut Ausonius (UMR 5607) \\ Maison de l'archéologie \\ 8, esplanade des Antilles \\ 33607 Pessac \\ sophie.gotteland(at)free.fr
}

